\title{
Flat adenomas exist in asymptomatic people: important implications for colorectal cancer screening programmes
}

\author{
A R Hart, S Kudo, E H Mackay, J F Mayberry, W S Atkin
}

\begin{abstract}
Background-Flat adenomas are nonexophytic with a flat top or central depression and histologically the depth of dysplastic tissue is never more than twice the mucosal thickness. Flat adenomas frequently contain severely dysplastic tissue, and may progress rapidly through the adenoma-carcinoma sequence. Flat lesions have never been described in a British asymptomatic population.

Aims-To determine whether flat adenomas exist in an asymptomatic population participating in a large randomised controlled trial of flexible sigmoidoscopy screening.

Patients-A total of 3000 subjects (aged 55-64 years) underwent screening by flexible sigmoidoscopy.

Methods-All polyps were removed and sent for histology. The number of polyps with endoscopic and histological features of flat adenomas was recorded.

Results-Three subjects had a total of four flat lesions-that is, one per 1000 people screened. Three contained severely dysplastic tissue, one a focus of adenocarcinoma. Three of the four lesions were less than $5 \mathrm{~mm}$ in size and the fourth was 15 $\mathrm{mm}$ in diameter.

Conclusions-Flat lesions with severe dysplasia exist in the asymptomatic population. This has major implications for gastroenterologists who should be trained to identify them. Their existence is of importance to molecular biologists and epidemiologists investigating the aetiology of colorectal cancer.

(Gut 1998;43:229-231)
\end{abstract}

Keywords: flat adenomas; screening; flexible sigmoidoscopy

The classic pattern of the development of colorectal cancer is from benign sessile or pedunculated adenomas. The adenomacarcinoma sequence theory is supported by histological evidence showing that a proportion of macroscopic adenomas contain malignant tissue, ${ }^{1}$ radiological data showing progressive enlargement of polyps, ${ }^{2}$ and cell culture work. ${ }^{3}$ Recent work on "flat adenomas" in Japan reported that such lesions were more likely to contain carcinoma ${ }^{4}$ or severe dysplasia than sessile adenomas of a similar size. ${ }^{5}$ Flat lesions can be defined endoscopically and histologically. Histologically, the dysplastic tissue does not protrude above the mucosal surface (flat depressed) or the dysplastic tissue is no more than twice the thickness of the mucosa (flat elevated). Radial extension of the dysplastic epithelium in the superficial mucosa without vertical extension to the crypt bases can also be shown. ${ }^{56}$ Endoscopically they are usually less than $1 \mathrm{~cm}$, and have a non-exophytic flat topped shape, sometimes with a central red coloured depression. ${ }^{4}$ Flat lesions have been described outside Japan, including in Germany $^{7}$ and in symptomatic patients in the United Kingdom. ${ }^{8}$

Previously, flat lesions have not been described in the asymptomatic British population. To see whether they exist in healthy people, we looked for flat adenomas in subjects attending for screening with flexible sigmoidoscopy in Leicestershire. The use of flexible sigmoidoscopy for screening is currently being evaluated by the Imperial Cancer Research Fund (ICRF) and Medical Research Council (MRC) in a large randomised controlled trial ${ }^{9}$ of which Leicester General Hospital is the first of the 13 large trial centres. The trial endoscopist spent a short time in an endoscopy unit in Japan learning to detect flat lesions. To help in their detection, it is essential that the bowel preparation is excellent and the mucosa is examined carefully.

\section{Methods}

During an 18 month period, 3000 asymptomatic people (aged 55-64 years) underwent flexible sigmoidoscopy as a screening test for colorectal cancer and adenomas in the ICRF/ MRC randomised controlled trial. These subjects were randomly chosen from a cohort of 13000 people who had expressed an interest in screening in Leicestershire. Bowel preparation was with either a single sachet of Picolax or one Fletcher's enema administered by the subject at home. Before the procedure, patients gave informed consent for both flexible sigmoidoscopy and polypectomy. All endoscopies were performed without sedation by a senior registrar in gastroenterology (ARH). Polyps less than $1 \mathrm{~cm}$ in diameter were removed at flexible sigmoidoscopy by hot biopsy or snare polypectomy. Subjects with lesions $1 \mathrm{~cm}$ or more in size were referred for colonoscopy, at which polypectomy was performed and the proximal colon examined. Patients with three or more adenomas, or an adenoma with severe dysplasia, or tubulovillous or villous architecture were also referred for colonoscopy. The detection rate for flat adenomas can be increased by dye 
Table 1 Characteristics of patients with endoscopic flat adenomas

\begin{tabular}{|c|c|c|c|c|}
\hline Patient, sex, age (y) & No of lesions & Location & Size $(\mathrm{mm})$ & Histology \\
\hline Patient A, female, 58 & 1 & Sigmoid & 3 & Tubular adenoma, mild dysplasia \\
\hline Patient B, male, 60 & 2 & Sigmoid & Both 4 & Both severely dysplastic tubular adenomas \\
\hline Patient C, male, 61 & 1 & Rectum & 15 & $\begin{array}{l}\text { Severely dysplastic tubulovillous adenoma with focus of } \\
\text { adenocarcinoma }\end{array}$ \\
\hline
\end{tabular}

Patients A and B were treated simply by cold biopsy. The polyp in patient C was treated by TART.

spraying and magnifying endoscopes, ${ }^{5}$ although this equipment was not available locally. The size, location, histology, and degree of dysplasia of each lesion was recorded and all adenomas were assessed histologically by a single pathologist (EHM).

\section{Results}

Three screenees (two males, one female) had endoscopically flat lesions within reach of the flexible sigmoidoscope which were confirmed histologically. Three of the lesions were in the sigmoid and one was in the rectum. No adenomas were found which looked endoscopically flat but which were histologically non-flat. Two patients had one flat lesion and the third had two. Three of the four lesions contained severe dysplasia, one with a focus of adenocarcinoma. Three of the adenomas were less than $5 \mathrm{~mm}$ in diameter, and the fourth was $15 \mathrm{~mm}$ in diameter. Table 1 and fig 1 show the characteristics of these patients and the endoscopic appearances of the lesions. Figure 2 shows the low power histological appearance of the flat lesion from patient $\mathrm{C}$ which showed severe dysplasia with a focus of carcinoma. The detection rate for polypoid adenomas in the first 1000 patients from this series was $8.6 \% .^{10}$

The three smaller adenomas were removed by cold biopsy at flexible sigmoidoscopy, while
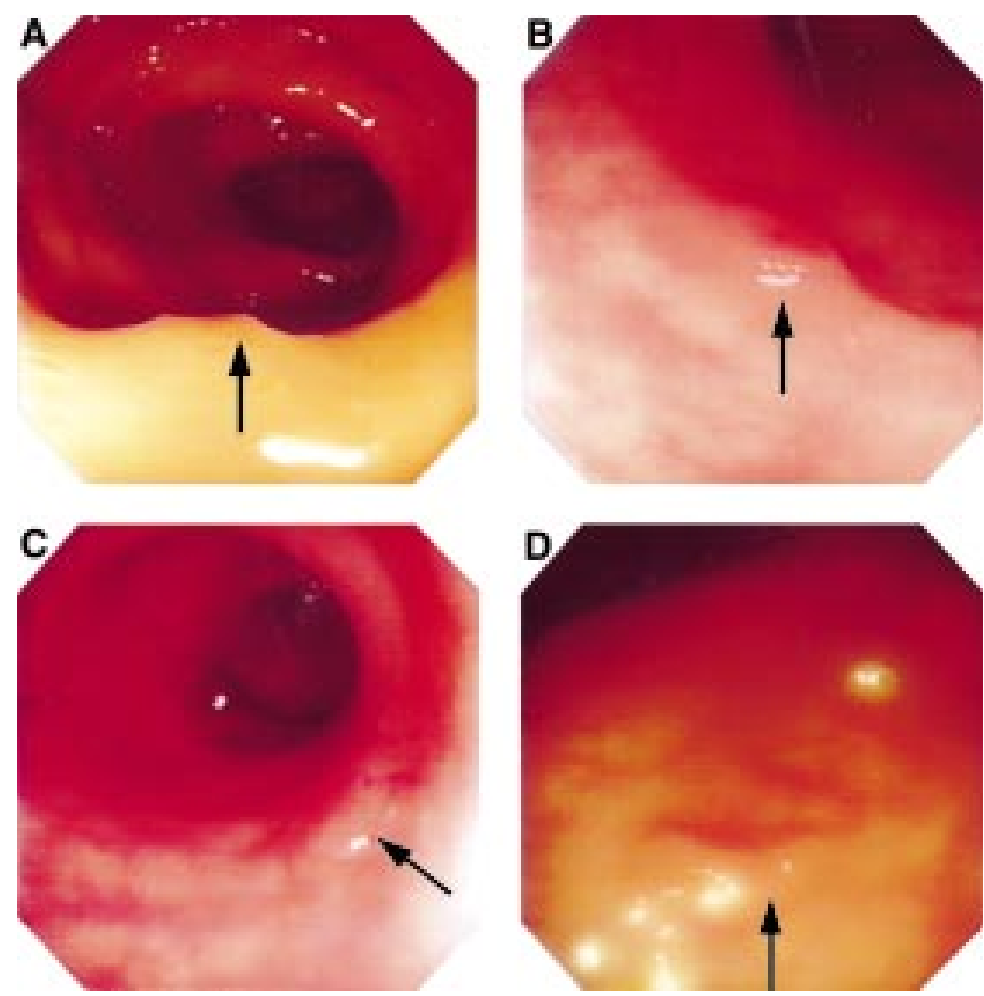

Figure 1 Endoscopic appearance of flat lesions from patient $A(A)$, patient $B(B, C)$, and patient $C$ (D).

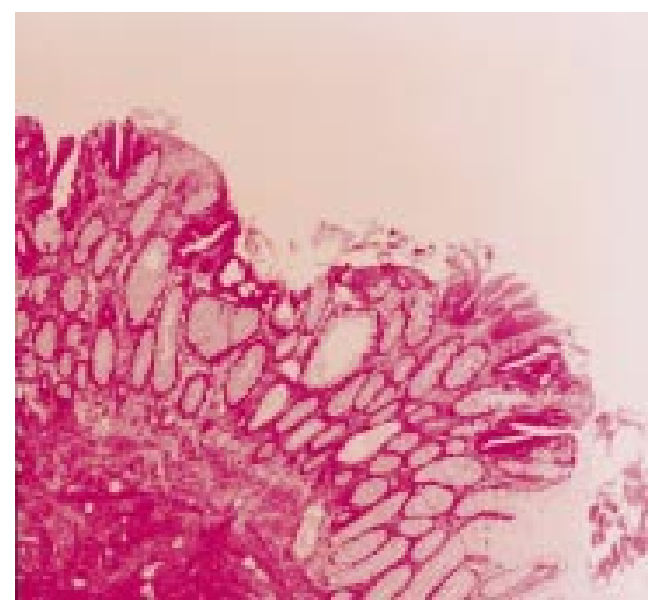

Figure 2 Low power view of histology from patient $C$.

the larger lesion required transanal resection (TART). The two patients with severely dysplastic lesions underwent colonoscopy, both of which were normal (in these patients the initial flat lesions had been completely removed when colonoscopy was performed). Periodic review of the site which contained carcinoma at three, six, and 12 months has not shown any recurrence.

\section{Discussion}

This is the first report of the existence of flat adenomas in a British asymptomatic population. The discovery has important implications for clinical gastroenterology and for scientists investigating the aetiology of colorectal cancer.

Gastroenterologists must now expand colonoscopy training programmes to educate clinicians to recognise and remove flat adenomas. Flat adenomas are clinically important as they are often severely dysplastic, a predictor of malignant potential. In our series, severe dysplasia was found in three quarters and one contained a focus of carcinoma. Identification of flat adenomas is difficult, because of their small size and therefore we may be underestimating their prevalence. Identification is aided by magnifying endoscopy and contrast chromoscopy techniques, but this equipment was unavailable locally. The magnifying endoscope has a magnifying capacity 40 times greater than that of conventional colonoscopes which allows the operator to examine small suspicious areas in greater detail. Spraying the lesion with an indigo carmine dye accentuates the contours of the lesion and margins and highlights the "pit pattern" of the crypts of Lieberkühn, allowing an estimation of histology. However, dye spraying and magnification would increase the duration of the procedure. Centres with a particular interest in colonoscopy should now assess the clinical role of this new technology. 
Flat adenomas are of interest to epidemiologists and molecular biologists investigating the aetiology of colorectal cancer. Such lesions frequently contain severely dysplastic tissue and are more likely to contain abnormalities of the p53 protein than polypoid lesions. ${ }^{11}$ The p53 gene, on chromosome 17 , produces a protein that slows the rate of the cell cycle, allowing time for DNA repair. Abnormalities of p53 occur late in carcinogenesis and may be an important step allowing cells to escape their normal controls on growth and become cancerous. More work is required to characterise the genetic characteristics of flat lesions and their relation to epidemiological factors such as diet.

Flat adenomas and carcinomas have been detected in only a few, mainly Japanese centres. $^{4-812}$ Kudo et al described a particular type of non-polypoid lesion known as a "depressed" lesion, where the tumour surface is lower than the adjacent normal mucosa. ${ }^{4}$ Most depressed lesions are less than $10 \mathrm{~mm}$ and are found in about one in every 140 Japanese patients. Importantly, in depressed lesions $6-10 \mathrm{~mm}$ in size, $50 \%$ had malignant tissue invading the submucosa. A group from Tokyo and Kobe City found severe atypia in $18 \%$ of the flat depressed type, compared with only $1.3 \%$ of diminutive polypoid adenomas. ${ }^{5}$ The Leeds Group, the only other British centre to describe flat adenomas, reported that $22.7 \%$ of patients attending for routine colonoscopy had an adenoma or carcinoma, of which $40 \%$ were morphologically flat or depressed. ${ }^{8}$ Flat lesions have also been found in Germany ${ }^{7}$ and Canada $^{12}$ and in the latter were 10 times more likely to contain severe dysplasia than were classic exophytic polyps of a similar size.
This paper described adenomas which were both endoscopically and histologically flat. However, the trial pathologist noted that some small endoscopically sessile (non-flat) lesions contained dysplastic tissue confined to the superficial part of the glandular crypts - that is, with the histological definition of flat adenomas. Detailed work is now on going in Leicester to identify and document the characteristics of these particular lesions.

Flat adenomas exist in the asymptomatic British population. An increased awareness of their importance should help prevent cancer and lead to a better understanding of its aetiology.

1 Muto T, Bussey HJR, Morson BC. The evolution of cancer of the colon and rectum. Cancer 1975;36:2251-70.

2 Stryker SJ, Wolff BG, Culp CE, et al. Natural history of untreated colonic polyps. Gastroenterology 1987;93:100913.

3 Williams AC, Harper SJ, Paraskeva C. Neoplastic transformation of a human colonic epithelial cell line: in vitro evidence for the adenoma to carcinoma sequence. Cancer Res 1990;50:4724-30

4 Kudo S, Tamura T, Nakajima S, et al. Depressed type of colorectal cancer. Endoscopy 1995;27:54-7.

5 Mitooka H, Fujimori T, Maeda S, et al. Minute flat depressed neoplastic lesions of the colon detected by contrast chromoscopy using an indigo carmine capsule.
trastic los trast chromoscopy using an indigc

6 Muto T, Kamiya J, Sawada T, et al. Small "flat adenoma" of Muto T, Kamiya J, Sawada T, et al. Small "flat adenoma"
the large bowel with special reference to its clinicopathological features. Dis Colon Rectum 1985;28:847-51.

7 Stolte M, Bethke B. Colorectal mini-de novo carcinoma: a reality in Germany too. Endoscopy 1995;27:286-90.

8 Rembacken BJ, Fujii T, Dixon M, et al. Flat and depressed colorectal neoplasia in England-are treatable cancers being missed [abstract]? Gut 1997;41(suppl 3):A58.

9 Atkin WS, Cuzick J, Northover JMA, et al. Prevention of colorectal cancer by "once-only" flexible sigmoidoscopy. Lancet 1993;341:736-40.

10 Hart AR, Mayberry JF, Mackay EH, et al. Results of the first 1000 patients from the pilot study for "once-only" flexible sigmoidoscopy screening for colorectal cancer and polyps [abstract]. Gut 1997;40:(suppl 1):A57.

11 Cawkwell L, Kennedy A, Dixon M, et al. p53 over expression in polypoid versus "flat" colorectal adenomas expression in polypoid versus "flat" co

12 Wolber RA, Owen DA. Flat adenomas of the colon. Hum Wolber RA, Owen DA.
Pathol 1991;22:70-4. 